\title{
Activation of direct and indirect pathways of glycogen synthesis by hepatic overexpression of protein targeting to glycogen
}

\author{
Robert M. O’Doherty, ${ }^{1}$ Per B. Jensen, ${ }^{1}$ Paul Anderson, ${ }^{1}$ John G. Jones, ${ }^{2}$ Hal K. Berman, ${ }^{1}$ \\ Denise Kearney, ${ }^{1}$ and Christopher B. Newgard ${ }^{1}$ \\ ${ }^{1}$ Gifford Laboratories for Diabetes Research, Marjorie Touchstone Diabetes Center; Departments of Biochemistry and \\ Internal Medicine; and \\ ${ }^{2}$ Mary Nell and Ralph B. Rogers Magnetic Resonance Center; University of Texas Southwestern Medical Center, Dallas, \\ Texas 75235 , USA
}

Address correspondence to: Christopher B. Newgard, Gifford Laboratories for Diabetes Research, Room Y8.212, University of Texas Southwestern Medical Center, 5323 Harry Hines Boulevard, Dallas, Texas 75235, USA.

Phone: (214)648-2930; Fax: (214) 648-9191; E-mail: newgard@utsw.swmed.edu.

Robert O'Doherty's current address is: University of Pittsburgh Medical Center, E1112 Biomedical Science Tower, Pittsburgh, Pennsylvania 15261, USA.

Received for publication October 14, 1999, and accepted in revised form January 4, 2000.

\begin{abstract}
Glycogen-targeting subunits of protein phosphatase-1, such as protein targeting to glycogen (PTG), direct the phosphatase to the glycogen particle, where it stimulates glycogenesis. We have investigated the metabolic impact of overexpressing PTG in liver of normal rats. After administration of PTG cDNA in a recombinant adenovirus, animals were fasted or allowed to continue feeding for 24 hours. Liver glycogen was nearly completely depleted in fasted control animals, whereas glycogen levels in fasted or fed PTG-overexpressing animals were 70\% higher than in fed controls. Nevertheless, transgenic animals regulated plasma glucose, triglycerides, FFAs, ketones, and insulin normally in the fasted and fed states. Fasted PTG-overexpressing animals receiving an oral bolus of $\left[\mathrm{U}-{ }^{13} \mathrm{C}\right]$ glucose exhibited a large increase in hepatic glycogen content and a $70 \%$ increase in incorporation of $\left[{ }^{13} \mathrm{C}\right]$ glucose into glycogen. However, incorporation of labeled glucose accounted for only a small portion of the glycogen synthesized in PTG-overexpressing animals, consistent with our earlier finding that PTG promotes glycogen synthesis from gluconeogenic precursors. We conclude that hepatic PTG overexpression activates both direct and indirect pathways of glycogen synthesis. Because of its ability to enhance glucose storage without affecting other metabolic indicators, the glycogen-targeting subunit may prove valuable in controlling blood glucose levels in diabetes.
\end{abstract}

J. Clin. Invest. 105:479-488 (2000).

\section{Introduction}

Hepatic glycogen stores are reduced in concert with an increase in net hepatic glucose production in all forms of human diabetes studied to date (1-3). Net hepatic glucose output is determined by the balance between the entry of glucose into storage and utilization pathways on the one hand and synthesis of glucose from the gluconeogenic and glycogenolytic pathways on the other. In non-insulin-dependent diabetes (NIDDM), the increase in hepatic glucose production occurs largely as a consequence of increased gluconeogenesis (1). However, the lower levels of liver glycogen in patients with NIDDM (1) indicate that the flux of glucose and gluconeogenic precursors into glycogen is impaired. This metabolic perturbation likely contributes to dysregulated hepatic glucose production in NIDDM and may be a site for therapeutic intervention.

Our group uses adenovirus-mediated gene transfer to investigate the metabolic impact of specific genes in isolated cells and in whole animals (4). This approach has been applied for the study of genes that influence hepatic glucose disposal and production. For example, overex-

pression of the catalytic subunit of glucose-6-phosphatase (G6Pase) increases hepatic glucose production and lowers glycogen content (5), whereas overexpression of glucokinase has the opposite effect (6). The latter studies suggest that maneuvers that increase the capacity of liver for glucose storage and utilization might be effective in lowering blood glucose in diabetes. In fact, hepatic overexpression of glucokinase largely normalizes glucose concentrations in streptozotocin-induced diabetic mice (7) and also significantly lowers glucose levels in ad libitum-fed normal rats and mice $(6,8)$. However, our work in normal rats demonstrates that levels of glucokinase overexpression sufficient for lowering of blood glucose have the undesired effect of increasing circulating concentrations of FFAs and triglycerides (6).

In the current study, we have investigated an alternative method for enhancing hepatic glucose disposal that focuses on improving the capacity for glycogen synthesis in liver. This has involved adenovirus-mediated delivery of protein targeting to glycogen (PTG), a member of the family of glycogen-targeting subunits of protein phosphatase-1 (PP-1). Glycogen-targeting subunits
\end{abstract}


bind to glycogen and to PP-1 and appear to facilitate regulation of enzymes of glycogen metabolism by the phosphatase (9-14). As found in this study and others $(10-12,15)$, at least 2 members of the glycogen-targeting subunit gene family, PTG and $\mathrm{G}_{\mathrm{L}}$, are expressed in normal liver. We have previously shown that overexpression of PTG in normal hepatocytes causes a dramatic enhancement in glycogen synthesis, in part via increasing the activity state of glycogen synthase (14). Conversely, streptozotocin-induced diabetes in rodents is associated with a decline in glycogen content that occurs in concert with a reduction in glycogen-targeting subunit expression in liver, without an effect on PP1 levels, suggesting a key role for the former protein in regulation of hepatic glycogen metabolism (15). The purpose of the current study was to gain further understanding of the regulatory potency of glycogen-targeting subunits by evaluating the metabolic impact of PTG overexpression in liver of normal rats.

\section{Methods}

Animal maintenance and administration of recombinant adenoviruses. All procedures were carried out in accordance with animal care guidelines of the University of Texas Southwestern Medical Center at Dallas and the National Research Council. Male Wistar rats (Charles River Laboratories, Wilmington, Massachusetts, USA) weighing 200-250 g were housed on an 0900-2100 hour light cycle and were allowed free access to water and standard laboratory chow (Harlan Teklad Laboratory, Winfield, Iowa, USA; $65 \%$ carbohydrate, $11 \%$ fat, $24 \%$ protein) unless otherwise specified. The rats were housed under these conditions for 1 week before adenovirus administration. Recombinant adenoviruses containing the cDNAs encoding mouse PTG (AdCMV-PTG) (14) or $\beta$-galactosidase (AdCMV- $\beta$ GAL) (16) were prepared, amplified, and purified as described previously (17). Between $0.5 \times 10^{12}$ and $1.0 \times 10^{12}$ recombinant adenovirus particles were administered via tail vein injection to rats anesthetized with a 50:5:1 mixture of ketamine (Avoco, Fort Dodge, Iowa, USA), Rompun (Avoco), and acepromazine (Haver, Shawnee, Kansas, USA), as described elsewhere $(6,18)$. After viral administration, animals were individually caged to allow monitoring of food intake and body weight before initiation of experiments.

Animal studies. Two experimental protocols were performed. In the first, animals were infused with either the AdCMV-PTG or AdCMV- $\beta$ GAL viruses. Ninety hours after virus administration, animals were either fasted for 24 hours or allowed to continue feeding ad libitum. Thereafter, all animals were anesthetized with Nembutal $(50 \mu \mathrm{g} / \mathrm{g}$ body weight intraperitoneally; Abbott Laboratories, North Chicago, Illinois, USA), blood samples were taken, and liver and skeletal muscle were excised and rapidly frozen in liquid nitrogen and stored at $-70^{\circ} \mathrm{C}$ until further analysis. In the second protocol, animals were infused with AdCMV-PTG or AdCMV- $\beta$ GAL. Ninety hours after virus administration, animals were fasted for 24 hours with free access to water before administration of a bolus of $\left[\mathrm{U}-{ }^{13} \mathrm{C}\right]$ glucose $(2 \mathrm{~g} / \mathrm{kg}$; enriched with $\left[{ }^{13} \mathrm{C}\right]$ glucose to $5 \%$ ) by gavage (oral glucose tolerance test [OGTT]). Blood samples (approximately $20 \mu \mathrm{L} / \mathrm{sample}$ from the tail vein, except for a $200-\mu \mathrm{L}$ sample at $180 \mathrm{~min}$ utes to allow insulin assay) were collected immediately before administration of the bolus and at 30, 60, 90, 120, 150 , and 180 minutes after the bolus to allow measurement of circulating glucose concentrations. Animals were killed immediately after the 180-minute time point for collection of liver, which was divided into 3 aliquots: 1 for measurement of PTG mRNA, 1 for measurement of glycogen content, and 1 for purification of glycogen to allow analysis of $\left[{ }^{13} \mathrm{C}\right]$ glucose incorporation (see later for analytical procedures).

Measurement of expression ofglycogen-targeting subunits in liver by semiquantitative multiplex RT-PCR. The procedure used was based on methods described previously by Jensen et al. (19). Total RNA was extracted from powdered liver tissue using RNeasy spin columns (QIAGEN Inc., Valencia, California, USA), following the manufacturer's instructions. First-strand cDNA was prepared using $1 \mu \mathrm{g}$ total RNA, the Superscript RT kit, and random hexamer primers (GIBCO BRL, Gaithersburg, Maryland, USA), according to the manufacturer's instructions. The cDNA was diluted 1:6 in distilled water, and PCR was carried out using $3 \mu \mathrm{L}$ of the diluted cDNA and a PCR mix containing Taq DNA polymerase (2.5 U) and buffer (Promega Corp., Madison, Wisconsin, USA), dNTP mix (final concentrations of $40 \mathrm{mM}$ of each dNTP, except dCTP, which was present at $20 \mathrm{mM}$ [GIBCO BRL]), and $1.25 \mu \mathrm{Ci}$ of [ $\alpha_{\left.-{ }^{33} \mathrm{P}\right]-\mathrm{dCTP}}(2,000$ $\mathrm{Ci} / \mathrm{mmol}$; Du Pont NEN Research Products, Boston, Massachusetts, USA) in a $25-\mu \mathrm{L}$ reaction volume, using a protocol provided by Promega. For studies of glycogen-targeting subunit expression, 3 primer sets (5 pmol of each primer) were included in each reaction, 1 set specific for mouse PTG, amplifying a 188-bp PCR fragment, the second set specific for rat $\mathrm{G}_{\mathrm{L}}$, amplifying a 232-bp PCR fragment, and the third set specific for elongation factor $-1 \alpha$ (EF-1 $\alpha$ ), amplifying a 201-bp PCR fragment, serving as the internal standard (see Table 1 for primer sequences and accession numbers). PCR conditions were an initial incubation at $95^{\circ} \mathrm{C}$ for 5-6 minutes, at which time the cDNA was added to the hot PCR mix ("hotstart" procedure). This was followed by 22 cycles of $94^{\circ} \mathrm{C}$ for 45 seconds, $55^{\circ} \mathrm{C}$ for 45 seconds, and $72^{\circ} \mathrm{C}$ for 45 seconds. The final PCR reactions were mixed with $98 \%$ formamide denaturing loading buffer and separated on a $6 \%(\mathrm{wt} / \mathrm{vol})$ polyacrylamide gel, containing $7 \mathrm{M}$ urea. The gel was subsequently dried and exposed to a phosphorimager screen, and the resulting scan was analyzed using ImageQuant from Molecular Dynamics (Sunnyvale, California, USA). Identical conditions and methods were used for studies of the expression of the gluconeogenic enzymes G6Pase (catalytic subunit), pyruvate carboxylase, and phosphoenolpyruvate carboxykinase, using primers shown in Table 1.

To be able to analyze specifically the expression of endogenous rat PTG, it was necessary to clone a fragment of its CDNA, as the rat PTG sequence is not known. Several primer sets to the mouse PTG sequence were gener- 
ated. One primer set was found to amplify rat PTG and resulted in the isolation and sequencing of a 334-bp fragment by standard techniques. This fragment corresponded to nucleotides 59-393 of the mouse PTG sequence (12). By aligning the mouse and rat PTG sequences, primer sets specific for the endogenous (rat) and transgene (mouse) forms of PTG were identified (Figure 1). These primer sets were chosen such that they amplified bands of different size (152 bp for rat PTG versus $188 \mathrm{bp}$ for mouse PTG), enabling unequivocal identification and quantification of the products. Endogenous $\mathrm{G}_{\mathrm{L}}$ could be detected at 22 cycles, but for quantitative analysis of endogenous $G_{L}$ and PTG in fasted and fed rats, multiplex-PCR was performed using 24 cycles and the procedures already described here. For studies of endogenous PTG and $\mathrm{G}_{\mathrm{L}}$ expression done at 24 cycles, TATA box binding protein (TBP) was used as the internal standard, using primers that amplify a 186-bp PCR fragment (Table 1).

The contribution of oral glucose to total glycogen synthesis analyzed by nuclear magnetic resonance spectroscopy. $\left[{ }^{13} \mathrm{C}\right]$ nuclear magnetic resonance (NMR) spectroscopy was used to quantify the excess ${ }^{13} \mathrm{C}$ enrichment in the glucosyl units of glycogen after oral administration of 5\%-enriched [U${ }^{13} \mathrm{C}_{6}$ ] glucose. Glycogen was purified from liver samples as described previously (20). The ratio of excess ${ }^{13} \mathrm{C}$ enrichment in glycogen to that of oral glucose was used to determine the fraction of glycogen synthesized from oral glucose. A total of 30-50 mg of purified glycogen was dispersed in $5 \mathrm{~mL}$ of $10 \mathrm{mM}$ sodium acetate buffer [ $\mathrm{pH} 4.5$ ] containing 20 units of amyloglucosidase. The sample was incubated at $55^{\circ} \mathrm{C}$ for 2 hours, resulting in complete hydrolysis of glycogen to glucose. The protein was precipitated by adding $0.5 \mathrm{~mL}$ of $70 \%$ perchloric acid, followed by centrifugation at $12,000 \mathrm{~g}$, and adjustment of the $\mathrm{pH}$ of the supernatant to 7.0 with $\mathrm{KOH}$. The insoluble potassium perchlorate was removed by centrifugation, and the supernatant was lyophilized and redissolved in $0.6 \mathrm{~mL} \mathrm{D}_{2} \mathrm{O}$ for NMR analysis. Proton decoupled $\left[{ }^{13} \mathrm{C}\right] \mathrm{NMR}$ spectra of extracts were obtained with a Varian Inova $14.1 \mathrm{~T}$ spectrometer (Palo Alto, California, USA) operating at $150.9 \mathrm{MHz}$ and equipped with a $5-\mathrm{mm}$ broadband probe. Spectra were acquired with 2,000 scans as described previously (21), and the free-induction decays were processed with $0.5 \mathrm{~Hz}$ exponential multiplication before Fourier transformation. The areas of ${ }^{13} \mathrm{C}$ resonances were quantified by a curve-fitting program (NUTS; Acorn NMR, Fremont, California, USA). Excess ${ }^{13} \mathrm{C}$ enrichment of glucose was measured from the $\left[{ }^{13} \mathrm{C}\right]$ NMR spectrum of carbon 1 using the method of Brainard et al. (22). The singlet resonance was assumed to reflect the natural abundance of ${ }^{13} \mathrm{C}$ at $1.1 \%$ enrichment, and the multiplets arising from ${ }^{13} \mathrm{C}-{ }^{13} \mathrm{C}$ spin-spin coupling reflect the excess enrichment from $\left[\mathrm{U}_{-}{ }^{13} \mathrm{C}_{6}\right] \mathrm{glu}-$ cose and from $\left[1,2-{ }^{13} \mathrm{C}_{2}\right]$ and $\left[1,2,3-{ }^{13} \mathrm{C}_{3}\right]$ glucose. The partially labeled glucose molecules are generated from gly- colysis of $\left[\mathrm{U}_{-}{ }^{13} \mathrm{C}_{6}\right]$ glucose to $\left[\mathrm{U}_{-}{ }^{13} \mathrm{C}_{3}\right]$ pyruvate followed by gluconeogenic incorporation of $\left[\mathrm{U}_{-}{ }^{13} \mathrm{C}_{3}\right]$ pyruvate into glycogen (23). Therefore, the $\left[{ }^{13} \mathrm{C} 1\right]$ multiplet represents the incorporation of oral $\left[\mathrm{U}-{ }^{13} \mathrm{C}\right]$ glucose into hepatic glycogen by both direct and gluconeogenic pathways. Calculation of the amount of $\left[{ }^{13} \mathrm{C}\right]$ glucose incorporated into glycogen was made as follows: $\left[{ }^{13} \mathrm{C}\right]$ glucose incorporation into glycogen $=\left(\right.$ area of ${ }^{13} \mathrm{C} 1$-multiplet/area of ${ }^{13} \mathrm{C} 1$-singlet $) \times 0.011$. The percentage of glycogen originating from oral glucose (\% total) was calculated as: \% total = excess enrichment glycogen $\mathrm{C} 1$ /excess enrichment oral glucose C1. Finally, the absolute amount of glycogen originating from oral glucose was calculated as: $\%$ total $\times$ mass of glycogen synthesized during the OGTT.

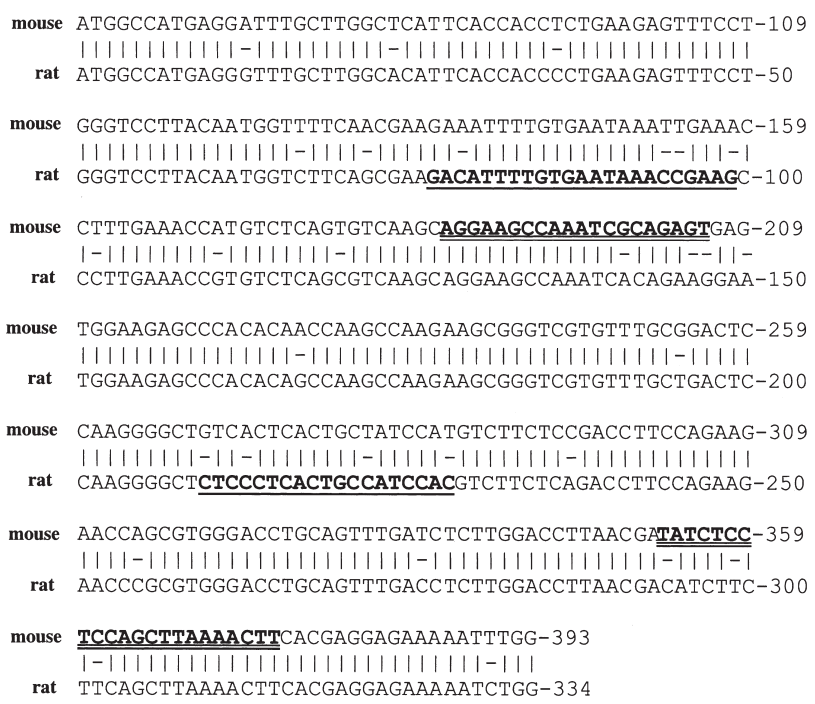

\section{Figure 1}

Alignment of the partial cDNA sequences of rat and mouse PTG. Vertical lines between the 2 sequences indicate identical nucleotides, and horizontal lines indicate nonidentical nucleotides. The oligonucleotide pairs used for amplification of endogenous rat PTG are shown in bold in the rat PTG sequence with a single underline. The oligonucleotide pairs used for amplification of the mouse PTG transgene are shown in bold in the mouse PTG sequence with a double underline. The numbering indicates that nucleotide 1 of the rat PTG sequence (cloned in this study) corresponds to nucleotide 59 of the published mouse PTG sequence (12). 
Plasma and tissue analysis. Plasma insulin levels were measured by RIA (Linco Research, St. Charles, Missouri, USA). Plasma triglycerides, FFAs, ketones, and lactate were measured using kits (Sigma Chemical Co., St. Louis, Missouri, USA). Plasma glucose was measured using a HemoCue Glucose Analyzer (HemoCue AB, Angelholm, Sweden). Glycogen content was measured by an amyloglucosidase-based assay, as described elsewhere (20).

Statistical analysis. Data are expressed as the mean \pm SEM. Statistical significance was determined by unpaired Student's $t$ test using the statistics module of Microsoft Excel (version 5.0; Microsoft Corp., Redmond, Washington, USA). Statistical significance was assumed at $P<0.05$.

\section{Results}

Expression of glycogen-targeting subunits in rat liver. In the first protocol of this study, animals were infused with either the AdCMV-PTG or AdCMV- $\beta$ GAL viruses. Ninety hours after virus administration, animals were either fasted for 24 hours or allowed to continue feeding ad libitum. Thereafter, blood samples were drawn and the animals were sacrificed for collection of liver and muscle samples. The first goal of this study was to evaluate expression of
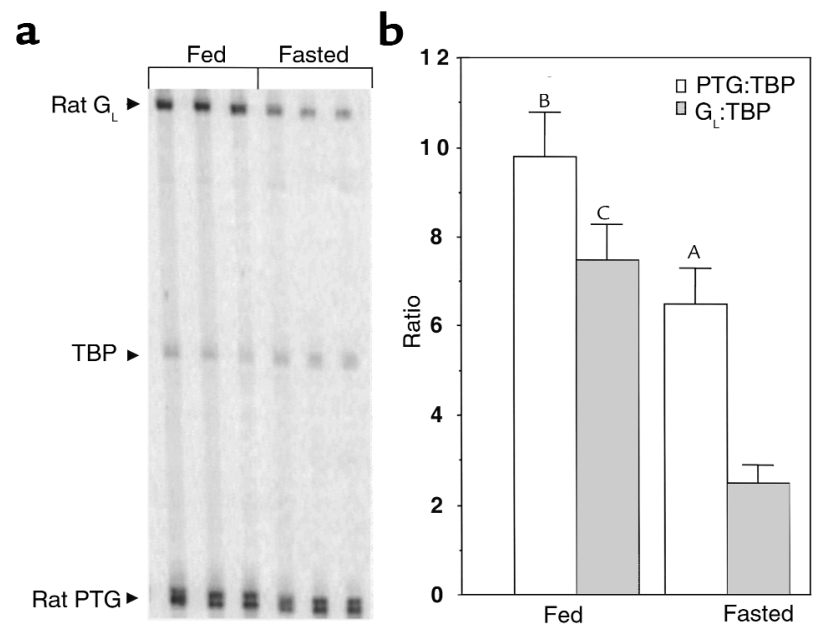

Figure 2

Expression of endogenous forms of glycogen-targeting subunits in liver of fasted and fed rats. Animals received either the AdCMV- $\beta G A L$ or AdCMV-PTG adenoviruses. Ninety hours after viral administration, animals were either allowed to continue feeding ad libitum or were fasted for 24 hours. Multiplex RT-PCR was performed on liver RNA samples from these animals to evaluate the levels of endogenous rat $G_{L}$ and rat PTG mRNA as function of fasting and feeding. Because the type of virus infused had no impact on endogenous $G_{L}$ or PTG expression, data from all fasted and fed animals were pooled. (a) Three representative samples for each group are displayed, showing amplification products for rat $G_{L}$, rat PTG, and the internal standard, TBP. (b) Quantitative analysis of the ratios of PTG/TBP and GL/TBP signals (see symbol legends in $\mathbf{b}$ ) was performed on all samples by exposing gels to a phosphorimager screen and processing the resulting scan with ImageQuant. Data represent the mean \pm SEM for 13 fed and 9 fasted animals. ${ }^{A}$ The PTG/TBP ratio in fasted animals was significantly higher than the $\mathrm{GL}_{L} / \mathrm{TBP}$ ratio in fasted animals $(P<0.001)$. ${ }^{\mathrm{B} T h e}$ ratio of PTG/TBP increased significantly in fed compared with fasted animals $(P=0.024)$. ${ }^{C}$ The ratio of $G_{L} /$ TBP increased significantly in fed compared with fasted animals $(P<0.001)$. endogenous and exogenous genes encoding glycogen-targeting subunits of PP- 1 in liver of fasted and fed rats. This was accomplished by a multiplex RT-PCR strategy performed in 2 phases. In the first phase, we evaluated the expression of the endogenous glycogen-targeting subunit genes, based on previous reports of hepatic expression of 2 members of the family, protein targeting to glycogen or PTG $(11,12)$ and $G_{L}(10,15)$. The expression of these 2 genes was measured semiquantitatively by simultaneous inclusion of oligonucleotide pairs specific for rat $\mathrm{G}_{\mathrm{L}}$, rat PTG, and an internal standard, TBP. Because only the sequences of mouse (12) and human (11) PTG were known, it was first necessary for us to clone a fragment of the rat PTG cDNA in order to identify unequivocally oligonucleotide primers that would be specific for the endogenous rat gene product. This was accomplished by RT-PCR, and an alignment of 334-bp fragments of the rat and mouse PTG sequences is presented in Figure 1. In the second phase, transgene expression was analyzed in RNA samples from the same fasted and fed rats analyzed in the first phase by inclusion of oligonucleotide pairs specific for mouse PTG (note that the AdCMV-PTG virus contains the cDNA encoding mouse PTG [14]) and, for standardization, $E F-1 \alpha$. The primers for rat $G_{L}$ were also included in this study to allow comparison of transgene expression with the level of 1 of the endogenous glycogen-targeting subunit gene products.

Expression of the endogenous gene products is summarized in Figure 2. Fasted rats expressed both PTG and $\mathrm{G}_{\mathrm{L}}$ in liver, but PTG expression was 2.6 times higher than $\mathrm{G}_{\mathrm{L}}$, when the 2 signals were normalized to the same internal standard. Analysis of liver samples from fed rats revealed a 50\% increase in PTG expression and a 300\% increase in $G_{L}$ expression relative to fasted animals. The relative expression levels of PTG and $\mathrm{G}_{\mathrm{L}}$ and the changes that occurred between fasted and fed states were not affected by the type of adenovirus that was administered, proving that the oligonucleotides used to amplify rat PTG did not cross-react with mouse PTG. Based on this finding, data from all fasted and fed animals were pooled to produce the results shown in Figure 2.

Expression of the mouse PTG transgene is shown in Figure 3. Note that we have previously shown that administration of recombinant viruses by systemic infusion into rats results in preferential expression of transgenes in liver, with no detectable expression in muscle, pancreas, brain, spleen, or adipose tissue, and very low levels of expression in lung $(5,6)$. These findings are similar to those reported for other rodent species (16). On the basis of these results, we have limited our analysis of transgene expression to liver in the current study.

Animals that received the AdCMV- $\beta$ GAL virus (fasted and fed) contained only background signal at the position expected for the amplified mouse PTG-derived product (188 bp). Of the 8 fasted rats that received the AdCMV-PTG virus, 5 exhibited a strong increase in PTG mRNA levels (high expressers), whereas 3 had lower levels of expression (low expressers). Of the 12 fed rats that received the AdCMV-PTG virus, 4 clearly overexpressed 
Figure 3

Expression of the mouse PTG transgene in liver, fasted/fed protocol. Animals received either the AdCMV- $\beta$ GAL or AdCMV-PTG adenoviruses. Ninety hours after viral administration, animals were either allowed to continue feeding ad libitum or were fasted for 24 hours. Multiplex RTPCR was performed on liver RNA samples from these animals. Inspection of the data revealed that AdCMV-PTG-treated animals could be segregated into high-expresser (high) and low-expresser (low) groups. (a) Three representative samples for each group are displayed, showing amplification products for the mouse PTG transgene and the internal standard EF- $1 \alpha$. (b) Quantitative analysis of the ratio of PTG/EF- $1 \alpha$ signals was performed on all samples by exposing gels to a phosphorimager screen, and processing the resulting scan with ImageQuant. Data represent the mean \pm SEM for the following number of samples: fed PTG high expressers, $n=4$; fed PTG low expressers, $n=8$; fed $\beta G A L$ controls, $n=6$; fasted PTG high expressers, $n=5$; fasted PTG low expressers, $n=$ 3 ; fasted $\beta G A L$ controls, $n=5$. ${ }^{A}$ Values were greater than in the $\beta G A L$ control group $(P<0.001)$. ${ }^{B}$ Values were greater in PTG high expressers than in PTG low expressers $(P<0.001)$.

the gene (high expressers), and 8 contained low levels of mouse PTG in liver (low expressers). Figure 3a shows 3 representative PCR samples from each of the aforementioned groups (fed and fasted AdCMV- $\beta$ GAL-treated animals, AdCMV-PTG-treated low expressers, and AdCMVPTG-treated high expressers). Scanning of all samples from each group revealed a mean increase in PTG/EF-1 $\alpha$ signal intensity of 39 -fold in the 5 fasted high expressers, and 34 -fold in the 4 fed high-expresser rats, relative to the background signal at the same position in samples from fasted and fed AdCMV- $\beta$ GAL-treated controls, respectively (Figure $3 \mathrm{~b}$ ). The fasted high expressers contained 3.5 times more PTG signal than fasted low expressers, whereas the fed high expressers had 15.7 times more PTG signal than fed low expressers. Finally, in the fed high expressers, levels of PTG mRNA exceeded the levels of endogenous $\mathrm{G}_{\mathrm{L}}$ by approximately 12.5 -fold, indicating that the transgene was the dominant form of glycogentargeting subunit present in high-expresser animals (data not shown). The same pattern of PTG expression was confirmed by RNAse protection analysis on a subset of the RNA samples (data not shown).

Effects of PTG overexpression on liver glycogen in fasted and fed rats. We have previously reported that overexpression of PTG in hepatocytes isolated from fasted rats causes a large increase in glycogen accumulation, even when these cells are cultured in the absence of glucose (14). We also showed that activation of glycogenolysis by agents such as forskolin and glucagon was impaired in PTG-overexpressing hepatocytes (14). We therefore investigated the effect of PTG overexpression on liver glycogen metabolism in fed and fasted rats.

As shown in Figure 4, AdCMV- $\beta$ GAL-infused rats contained high levels of liver glycogen when allowed to feed ad libitum $(156 \pm 15 \mu \mathrm{g}$ glycogen per milligram of protein), whereas liver glycogen was almost completely depleted by fasting of this control group for 24 hours, the expected pattern for normal animals (20). In sharp contrast, animals with clear hepatic overexpression of PTG (high expressers) experienced no depletion of hepatic glycogen stores during fasting. Interestingly, fed and fast-

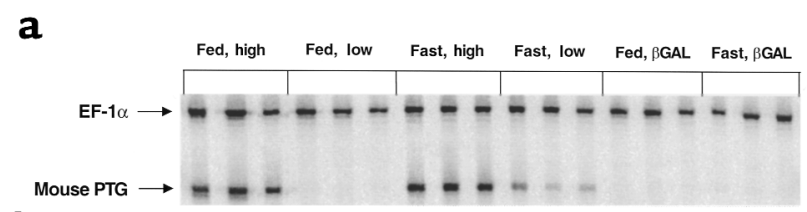

b

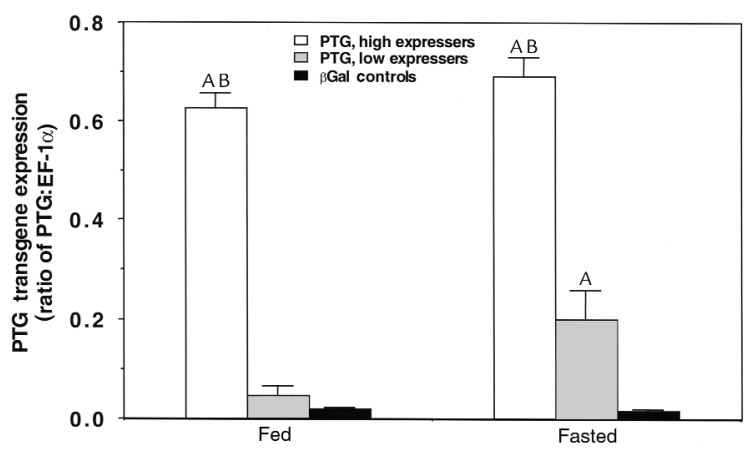

ed high overexpressers had nearly identical hepatic glycogen levels $(257 \pm 32$ and $249 \pm 35 \mu \mathrm{g}$ glycogen per milligram protein, respectively), these values being $70 \%$ higher than in liver of fed control animals $(P=0.015)$. These effects seemed to require a threshold of PTG overexpression, as animals that were treated with AdCMV-PTG but that were low expressers based on the analysis shown in Figure 3 experienced no increase in glycogen content in the fed state and a modest increase in the fasted state relative to AdCMV- $\beta$ GAL controls. Muscle glycogen was also measured. Levels of $32.1 \pm 2.1,32.1 \pm 1.5$, and $37.3 \pm$ $3.5 \mu \mathrm{g}$ glycogen per milligram of protein were measured in high expressers, low expressers, and AdCMVBGAL-treated fed rats, respectively, and 17.1, 21.5, and

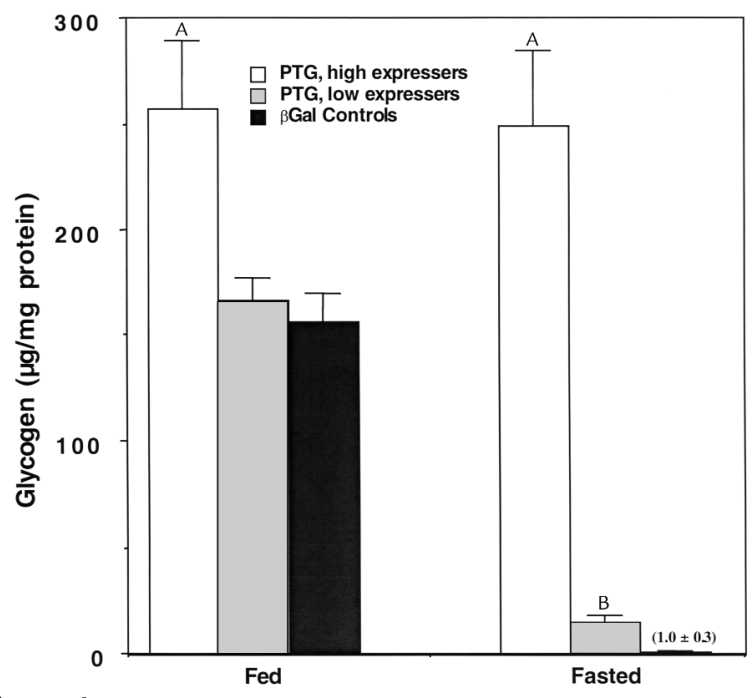

Figure 4

Liver glycogen levels in fasted and ad libitum-fed rats. Animals were treated as described in the legend to Figure 3, and liver samples were used for measurement of glycogen content. Data represent the mean \pm SEM, and the experimental groups and the number of animals in each group are as described in the legend to Figure 3. A Liver glycogen was significantly higher in the PTG high expressers than in either the PTG low expressers or $\beta G A L$ controls $(P<0.001)$. ${ }^{B}$ Liver glycogen was significantly higher in the fasted PTG low-expresser group than in the fasted $\beta G A L$ control group $(P<0.001)$. 
$19.7 \mu \mathrm{g} / \mathrm{mg}$ protein in high expressers, low expressers, and AdCMV- $\beta$ GAL-fasted rats, respectively. These data demonstrate that PTG overexpression in liver was without effect on muscle glycogen metabolism and did not divert glucose storage from the periphery to the liver.

PTG overexpression does not perturb homeostasis of key circulating fuels. We have previously shown that adenovirusmediated overexpression of glucokinase in liver results in lowered blood glucose concentrations and increased hepatic glycogen storage, but also undesired increases in circulating FFAs and triglycerides (TGs) (6). We therefore investigated whether PTG overexpression caused any changes in circulating metabolite and hormone levels in the same fasted and fed animals analyzed in the foregoing figures. As shown in Table 2, normal changes in serum variables were noted in PTG high expressers compared with either PTG low expressers or AdCMV$\beta G A L-i n f u s e d$ control animals in response to fasting and feeding. Thus, plasma glucose, TG, and insulin were lowered in all groups of fasted animals, whereas FFA and ketones were increased. Neither the type of virus admin-

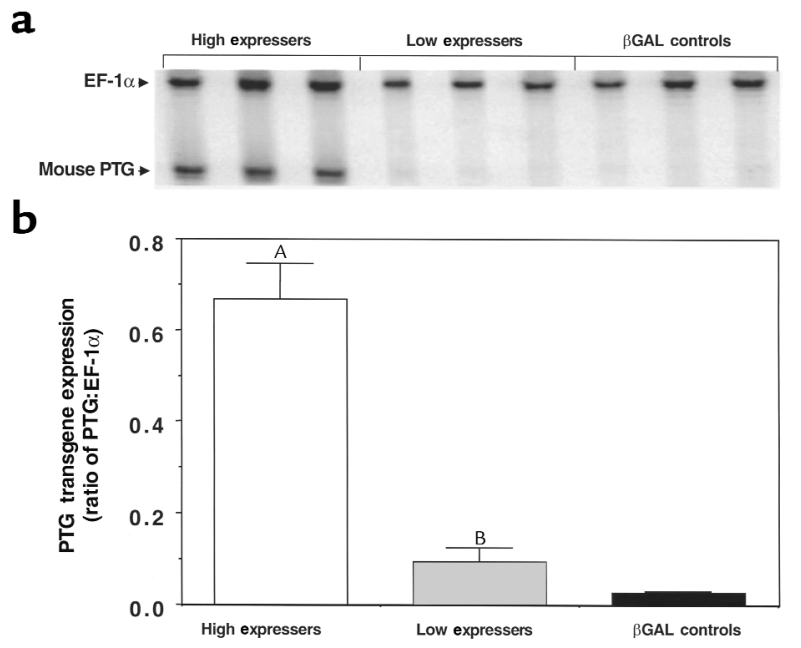

Figure 5

Expression of the mouse PTG transgene in liver, OGTT protocol. Animals received either the AdCMV-PTG or AdCMV- $\beta$ GAL adenoviruses and were allowed to feed ad libitum for 90 hours after viral administration. Animals were then fasted for 24 hours before receiving an oral bolus of $\left[{ }^{13} \mathrm{C}\right]$-labeled glucose $(2 \mathrm{~g} / \mathrm{kg})$. Animals were sacrificed for collection of liver samples 180 minutes after the oral glucose challenge, and a portion of these samples was used for preparation of RNA and multiplex RT-PCR analysis as described in the legend to Figure 3. Inspection of the data revealed that AdCMV-PTG-treated animals could be segregated into high-expresser and low-expresser groups. (a) Three representative samples for each group are displayed, showing amplification products for the mouse PTG transgene and the internal standard, EF-1 $\alpha$. (b) Quantitative analysis of the ratio of PTG/EF-1 $\alpha$ signals was performed on all samples by exposing gels to a phosphorimager screen and processing the resulting scan with ImageQuant. Data represent the mean \pm SEM for the following number of samples: PTG high expressers, $n=9$; PTG low expressers, $n=7 ; \beta G A L$ controls, $n=9$. APTG was expressed at a higher level in the high expressers than in either the low expressers or $\beta G A L$ controls $(P<0.001)$. ${ }^{\text {BT }}$ The values were greater in PTG low expressers than in $\beta G A L$ controls $(P=0.023)$. istered nor the extent of PTG overexpression had any significant effect on these metabolic variables in either the fasted or fed states. Thus, PTG overexpression in liver enhances hepatic glucose clearance and storage without affecting other key metabolic indicators.

OGTT in normal rats with adenovirus-mediated PTG overexpression in liver. Although no obvious changes in blood glucose levels were detected in fasted or ad libitum-fed rats with overexpressed PTG in liver (Table 2), the large increase in glycogen storage in these animals suggested that disposal of glucose during an OGTT might be altered. To test this idea, normal rats received either the AdCMV- $\beta$ GAL or AdCMV-PTG viruses by systemic infusion. After 90 hours of ad libitum feeding, the animals were fasted for a period of 24 hours before administration of a bolus of ${ }^{13} \mathrm{C}$-enriched glucose $(2 \mathrm{~g} / \mathrm{kg}$; enriched with $\left[{ }^{13} \mathrm{C}\right]$ glucose to $5 \%$ ) by gavage. At 180 minutes after the oral glucose bolus, animals were sacrificed for collection of the liver, which was aliquoted into 3 portions: 1 for analysis of PTG mRNA, 1 for analysis of glycogen content, and 1 for purification of liver glycogen to allow NMR analysis of $\left[{ }^{13} \mathrm{C}\right]$ glucose incorporation into glycogen.

Levels of PTG RNA were analyzed in animals that received the AdCMV- $\beta$ GAL or AdCMV-PTG adenoviruses using the multiplex RT-PCR assay. Animals that received AdCMV- $\beta$ GAL did not express the mouse PTG transgene product. Similar to the first protocol, $9(56 \%)$ of 16 animals treated with AdCMV-PTG showed clear evidence of expression of PTG RNA, whereas the other 7 animals showed either no detectable overexpression of the transgene or a modest increase (representative samples are shown in Figure 5a). Scanning of samples from all animals in the OGTT study revealed 25-fold and 7fold overexpression of PTG in high expressers relative to the background signal in samples from AdCMV$\beta G A L-t r e a t e d$ animals, and the low signal in AdCMVPTG low expressers, respectively (Figure $5 \mathrm{~b}$ ). Based on the findings of negligible metabolic effects of low levels of PTG overexpression in the first protocol of this study, the AdCMV- $\beta$ GAL-treated and AdCMV-PTG low expressers were pooled as the control group for the second protocol (OGTT). Because the samples analyzed in Figures 3 and 5 were amplified at the same time, run on the same gel, and normalized against the same internal standard (EF-1 $\alpha$ ), we can conclude that levels of PTG overexpression were similar in the high-expresser groups of fasted, fed, and OGTT animals.

Figure 6 shows the results of the OGTT in animals with and without strong PTG overexpression in liver. Immediately before administration of the glucose bolus (time 0 ), the fasting blood glucose level in the PTG high expressers was lower than the controls, although this difference did not reach statistical significance $(P=0.056)$. This trend toward lower blood glucose concentrations in PTG-overexpressing animals became more evident during the OGTT and reached statistical significance at 30, 90,150 , and 180 minutes after administration of the glucose bolus ( $P$ values: $0.005-0.03$ ). The differences between PTG-overexpressing and control animals at 60 and 120 
Plasma variables in fasted and fed AdCMV-PTG and AdCMV- $\beta$ GAL-treated rats

\begin{tabular}{|c|c|c|c|c|c|c|c|}
\hline Animal group & $\begin{array}{l}\text { Glucose } \\
(\mathrm{mg} / \mathrm{dL})\end{array}$ & $\begin{array}{l}\text { Triglycerides } \\
(\mathrm{mg} / \mathrm{dL})\end{array}$ & $\begin{array}{c}\text { FFAs } \\
(\mathrm{mmol} / \mathrm{L})\end{array}$ & $\begin{array}{l}\text { Ketones } \\
(\mathrm{mg} / \mathrm{dL})\end{array}$ & $\begin{array}{l}\text { Lactate } \\
(\mathrm{mg} / \mathrm{dL})\end{array}$ & $\begin{array}{l}\text { Insulin } \\
(\mathrm{ng} / \mathrm{mL})\end{array}$ & $\begin{array}{c}\text { Food intake } \\
(\mathrm{g} / 24 \mathrm{~h})\end{array}$ \\
\hline $\begin{array}{l}\text { Fed, AdCMV-PTG, } \\
\text { high expressers }(n=4)\end{array}$ & $215 \pm 30$ & $88 \pm 12$ & $0.10 \pm 0.02$ & $2.2 \pm 0.7$ & $23 \pm 54.2 \pm 0.6$ & $23 \pm 1$ & \\
\hline $\begin{array}{l}\text { Fed, AdCMV-PTG, } \\
\text { low expressers }(n=8)\end{array}$ & $216 \pm 9$ & $89 \pm 11$ & $0.08 \pm 0.01$ & $3.0 \pm 0.3$ & $36 \pm 4$ & $3.8 \pm 0.7$ & $23 \pm 1$ \\
\hline $\begin{array}{l}\text { Fasted, AdCMV-PTG, } \\
\text { high expressers }(n=5)\end{array}$ & $152 \pm 10$ & $23 \pm 3$ & $0.43 \pm 0.14$ & $14.2 \pm 2.5$ & $45 \pm 9$ & $0.4 \pm 0.1$ & 0 \\
\hline $\begin{array}{l}\text { Fasted, AdCMV-PTG, } \\
\text { low expressers }(n=3)\end{array}$ & $138 \pm 15$ & $26 \pm 7$ & $0.23 \pm 0.03$ & $8.6 \pm 4.3$ & $29 \pm 6$ & $0.4 \pm 0.2$ & 0 \\
\hline $\begin{array}{l}\text { Fed, AdCMV- } \beta \text { GAL } \\
(n=6)\end{array}$ & $214 \pm 6$ & $88 \pm 2$ & $0.08 \pm 0.01$ & $1.7 \pm 0.7$ & $40 \pm 8$ & $4.6 \pm 0.3$ & $27 \pm 1$ \\
\hline $\begin{array}{l}\text { Fasted, AdCMV- } \beta G A L \\
(n=5)\end{array}$ & $139 \pm 6$ & $24 \pm 6$ & $0.40 \pm 0.08$ & $16.0 \pm 3.0$ & $24 \pm 6$ & $0.3 \pm 0.1$ & 0 \\
\hline
\end{tabular}

Animals received either the AdCMV- $\beta G A L$ or AdCMV-PTG adenoviruses. Ninety hours after viral administration, animals were either allowed to continue feeding ad libitum or were fasted for 24 hours before blood sampling at approximately 1000 hours for analysis of the indicated plasma variables. The column labeled "Food intake" shows the weight of food consumed over the final 24 hours of the postviral infusion period. For the animals that received the AdCMVPTG virus, high- and low-expresser groups were identified as described in Figure 3. Data represent the mean \pm SEM for the number of animals indicated in each group. Although normal changes in serum variables were noted as a function of nutritional status in both AdCMV- $\beta$ GAL and AdCMV-PTG-treated animals, the type of virus administered or the extent of PTG overexpression had no significant effect on any of the measured metabolic indicators.

minutes also approached the significance threshold $(P=$ 0.068 and 0.062 , respectively). On the basis of these data, we conclude that PTG overexpression in liver of normal rats caused a modest enhancement in the disposal of an oral glucose load. We also measured plasma insulin at the 180-minute time point of the OGTT. The PTG highexpresser group had insulin levels of $0.27 \pm 0.08 \mathrm{ng} / \mathrm{mL}$, compared with $1.11 \pm 0.31 \mathrm{ng} / \mathrm{mL}$ in the controls $(P=$ $0.049)$. Note also that Table 1 showed similar insulin levels in PTG-overexpressing and control rats in the fasted or fed states, consistent with equal glucose levels in those animals. Thus, insulin seems to track with glucose in the PTG overexpressers, and the higher glycogen deposition during OGTT in these animals is made all the more remarkable by their relative hypoinsulinemia.

Liver glycogen levels after the OGTT. Control and PTGoverexpressing animals that received the oral glucose bolus were sacrificed at the conclusion of the test (180minute time point) for analysis of liver glycogen levels. Figure 7 shows that animals with hepatic PTG overexpression stored 7.6 times as much glycogen in liver as controls. Thus, the improved glucose tolerance in PTGoverexpressing animals shown in Figure 6 was accompanied by a dramatic increase in glycogen storage. Note also that the levels of glycogen stored in the PTG-overexpressing animals after the glucose bolus was 490 $\mu \mathrm{g} / \mathrm{mg}$ protein, nearly double the levels measured in PTG-overexpressing fasted or ad libitum-fed rats (compare Figures 4 and 7), providing a measure of the increment in glycogen storage during the glucose bolus.

$N M R$ analysis of the percentage of circulating glucose stored as liver glycogen. The oral glucose bolus was enriched with $\left[\mathrm{U}-{ }^{13} \mathrm{C}\right]$ glucose. This was done to enable evaluation of the incorporation of circulating glucose into liver glycogen during the OGTT in PTG-overexpressing and control rats. Glycogen was purified from a portion of livers excised from animals at the 180-minute time point after administration of the glucose bolus and was analyzed by $\left[{ }^{13} \mathrm{C}\right] \mathrm{NMR}$. As shown in Table 3, PTG-overexpressing animals incorporated $70 \%$ more $\left[{ }^{13} \mathrm{C}\right]$ glu- cose into glycogen than did control animals $(P=0.015$ for this comparison). However, as also shown in Table 3 , the percentage of liver glycogen derived from the oral glucose is much higher in the control group than in the PTG-overexpressing animals ( $19.5 \%$ in controls relative to $3.5 \%$ in the PTG-overexpressing animals). This difference is partially explained by the fact that the PTGoverexpressing animals contain much higher glycogen levels at the start of the OGTT than do controls (249 $\mu \mathrm{g} / \mathrm{mg}$ protein in fasted PTG high expressers versus $1-15 \mu \mathrm{g} / \mathrm{mg}$ in fasted controls; see Figure 4). However, the increment in glycogen synthesis that occurred during the OGTT was also 4.1 times higher in PTG-overexpressing animals than in controls $(490 \mu \mathrm{g} / \mathrm{mg}$ at end of OGTT in PTG-expressing animals $-249 \mu \mathrm{g} / \mathrm{mg}$ in fasted PTG-expressing animals $=241 \mu \mathrm{g} / \mathrm{mg} ; 62 \mu \mathrm{g} / \mathrm{mg}$ at the end of the OGTT in controls - average of 6.2

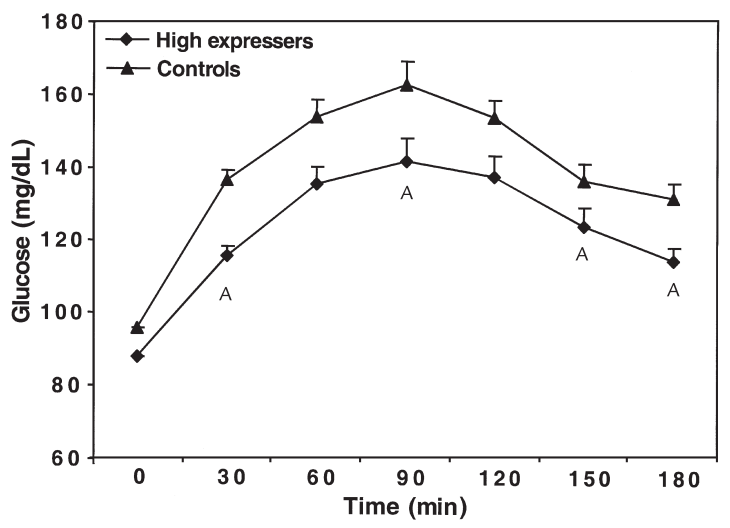

Figure 6

OGTT. Animals were treated as described in the legend to Figure 5. Plasma glucose concentrations were measured at the indicated times after the glucose bolus. The high-expresser group was as defined in Figure 5. The control group consisted of all AdCMV- $\beta G A L-i n f u s e d$ animals and AdCMV-PTG-infused low expressers as defined in Figure 5. Data represent the mean \pm SEM for 9 high expressers and 16 animals in the control group. AThose time points at which glucose levels in the PTG high expressers were lower than the control group ( $P$ ranging from 0.005 to 0.03 ). 
Table 3

Analysis of $\left[{ }^{13} \mathrm{C}\right]$ glucose incorporation into glycogen

\begin{tabular}{|c|c|c|c|c|}
\hline Experimental group & $\begin{array}{c}\text { A } \\
\text { Oral glucose incorporated } \\
\text { into glycogen }(\mu \mathrm{g} / \mathrm{mg} \text { protein })\end{array}$ & $\begin{array}{c}\text { B } \\
\text { \% Total glycogen from } \\
\text { oral glucose }\end{array}$ & $\begin{array}{c}\mathrm{C} \\
\text { Glycogen synthesis } \\
\text { during OGTT } \\
(\mu \mathrm{g} / \mathrm{mg} \text { protein })\end{array}$ & $\begin{array}{c}\text { D } \\
\% \text { New glycogen from } \\
\text { oral glucose }\end{array}$ \\
\hline $\begin{array}{l}\text { AdCMV-PTG, high } \\
\text { expressers }(n=9)\end{array}$ & $14.1 \pm 1.3^{\mathrm{A}}$ & $3.5 \pm 0.4$ & $241 \pm 69^{A}$ & 5.9 \\
\hline Controls $(n=16)$ & $8.2 \pm 1.2$ & $19.5 \pm 3.1^{\mathrm{A}}$ & $56 \pm 9$ & 14.6 \\
\hline
\end{tabular}

$\mu \mathrm{g} / \mathrm{mg}$ in fasted controls $=56 \mu \mathrm{g} / \mathrm{mg}$ ). This allows a calculation of the percentage of new glycogen formed from oral glucose during the OGTT. As shown in Table $3,5.9 \%$ of the new glycogen came from oral glucose in PTG-overexpressing animals compared with $14.6 \%$ in controls. These data strongly suggest that although PTG overexpression in liver modestly enhanced glucose incorporation into glycogen, its predominant effect was activation of glycogen synthesis from gluconeogenic precursors via the indirect pathway.

Expression of gluconeogenic enzymes in PTG-overexpressing animals. The NMR experiments suggest the possibility that PTG overexpression affects expression of key gluconeogenic enzymes. To evaluate this idea, we used the multiplex PCR approach to measure phosphoenolpyruvate carboxykinase (PEPCK), pyruvate carboxylase (PC), and G6Pase mRNA levels. After OGTT, PEPCK and PC expression were unchanged in liver of PTG-overexpressing animals compared with controls, but G6Pase expression was found to be reduced by $70 \%$

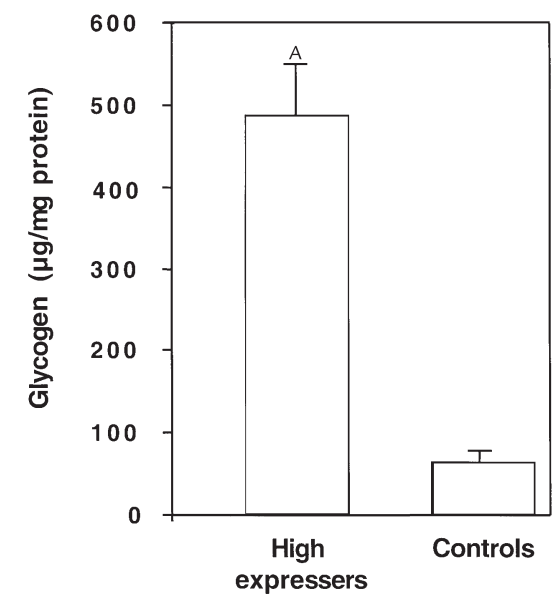

Figure 7

Liver glycogen levels after the OGTT. Animals were sacrificed for collection of liver samples at the 180-minute time point of the OGTT shown in Figure 6. Glycogen content was measured in the PTG high expressers and controls (see legends to Figure 5 and 6 for explanation). Data represent the mean \pm SEM for 9 PTG high expressers and

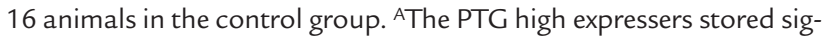
nificantly more liver glycogen than the controls $(P<0.001)$.
(Figure 8). This finding could help to explain the significant diversion of gluconeogenic carbon into glycogen (activation of the indirect pathway) as indicated by the $\left[{ }^{13} \mathrm{C}\right] \mathrm{NMR}$ measurements. However, this result does not readily explain how fasted PTG-overexpressing animals are able to retain low-normal blood glucose levels (see Figure 6 and Table 2). We have therefore also measured gluconeogenic enzyme expression in the fasted and ad libitum fed groups of animals that did not undergo OGTT. We find no significant changes in expression of gluconeogenic enzymes in PTG-overexpressing fed or fasted animals (including G6Pase), although G6Pase was reduced nonsignificantly in fasted PTG overexpressers. Thus, maintenance of low-normal glucose levels in fasted PTG-overexpressing rats is consistent with retention of near-normal levels of expression of gluconeogenic enzymes in these animals. During the OGTT, glucose appears to stimulate G6Pase gene expression effectively in control rats, but is less effective at this level in PTG-overexpressing animals. The effect of glucose to stimulate G6Pase expression in liver cells is well known (24-26). The mechanism by which glucose exerts this effect is not understood, but our new data suggest that PTG overexpression depletes a glucose-derived intermediate that may be essential for induction of G6Pase. Future experiments will be required to test this novel idea.

\section{Discussion}

In this study, we have investigated the metabolic impact of hepatic overexpression of PTG, a member of a family of glycogen-targeting subunits of PP-1. There were 2 major reasons for performing this work. First, we sought to determine whether the profound enhancement of glycogen accumulation and loss of regulation by glycogenolytic agents observed in PTG-overexpressing hepatocytes (14) would also occur upon expression of this protein in liver of intact animals. Second, we wanted to compare the metabolic impact of overexpression of PTG in liver with that of overexpressed glucokinase. We have previously shown that adenovirusmediated expression of glucokinase in liver of normal rats results in lowering of blood glucose and insulin lev- 
els in fed animals, but this is accompanied by a doubling of circulating TG and a 3-fold increase in FFA (6). Because some of this glucokinase-induced increase in circulating lipids could be explained by increased lipogenesis secondary to enhanced glycolytic flux, a hypothesis of the current work was that perturbation of lipid homeostasis might be avoided by using a protein such as PTG that preferentially diverts glucose into glycogen.

With regard to the first point, PTG overexpression in liver of intact animals had a profound impact on glycogen metabolism similar in many respects to its effects in isolated hepatocytes. Thus, PTG-expressing animals failed to deplete liver glycogen during a 24-hour fast despite the fact that glycogenolysis is known to be stimulated during fasting as a result of increases in circulating glucagon levels (27). Furthermore, expression of PTG in liver of normal rats dramatically increased glycogen levels after an oral glucose bolus relative to control animals. These results compare well with our studies on PTG-overexpressing hepatocytes, in which we noted an increase in glycogen synthesis in response to an increase in glucose concentration and very limited activation of glycogenolysis in response to the addition of glucagon or forskolin (14).

These effects seem to require a threshold of PTG overexpression. Thus, modest overexpression of PTG (lowexpresser groups) caused no increase in glycogen content in fed animals and only a very modest increase in fasted animals (see Figure 4). This finding is interesting when compared with changes that occur in the expression of endogenous glycogen-targeting subunits in liver. Figure 2 shows that the expression of PTG and $G_{L}$ is increased in fed compared with fasted animals. Our data on expression of $G_{L}$ in fasted and fed states are in agreement with a previous study of $\mathrm{G}_{\mathrm{L}}$ protein levels (15), although those investigators did not measure expression of PTG. PTG is the major form expressed in fasted rats, whereas $G_{L}$ undergoes the larger change in the fasted/fed transition. However, endogenous glycogen-targeting subunit expression represented only a small fraction of the transgene expression levels reached in the high-expresser groups of AdCMV-PTG-treated animals. These findings suggest that regulation of glycogen-targeting subunit expression may be an important event in activation of glycogen synthesis in the fasted/fed transition but that these changes are not sufficient to activate glycogenesis to full capacity.

Some insights into the mechanism of action of PTG are provided by our studies in which NMR was used to measure incorporation of $\left[{ }^{13} \mathrm{C}\right]$ glucose into glycogen. The finding that PTG overexpression caused a small but significant increase in $\left[{ }^{13} \mathrm{C}\right]$ glycogen labeling is consistent with the modest improvement in glucose disposal observed during the OGTT. Other studies have shown that overexpression of PTG causes activation of glycogen synthase $(12,14)$. We suggest that this action of the glycogen-targeting subunit enhances the conversion of glucose into glycogen and improves the efficiency of hepatic glucose disposal during a glucose

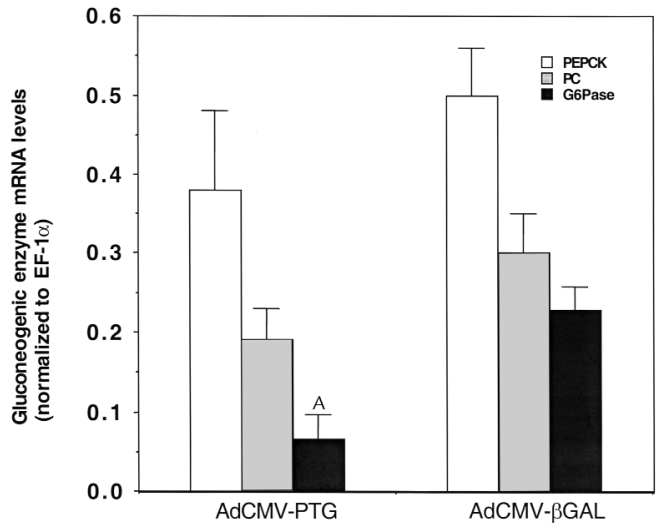

Figure 8

Effect of PTG on gluconeogenic enzyme expression. Levels of PEPCK, PC, and G6Pase mRNA were measured by multiplex PCR in liver samples from 4 PTG high expressers and 4 AdCMV- $\beta G A L$ infused controls (a subset of the samples used in Figure 5). Data were normalized to EF- $1 \alpha$ as the internal control. G6Pase expression was reduced by $70 \%$ in PTG high expressers compared with AdCMV- $\beta G A L$ controls ( ${ }^{A} P=0.01$ ), whereas expression of PEPCK and PC did not change significantly.

load. However, a striking finding of this study is that the direct incorporation of glucose into glycogen accounts for only a small portion of glycogen synthesized in liver in PTG-overexpressing animals during the OGTT. In normal rats in the fasted/refeeding transition, a significant portion of liver glycogen is synthesized by the so-called indirect pathway, in which gluconeogenic precursors coming from the circulation or residing within the liver serve as precursors $(20,28)$. The results of the current study suggest that overexpression of PTG enhances glycogen synthesis by this pathway, as only $5.9 \%$ of the glycogen synthesized during the OGTT in PTG-overexpressing animals was contributed by direct incorporation of oral glucose into glycogen. This is consistent with our finding in isolated hepatocytes in which potent activation of glycogen synthesis was observed even in the complete absence of carbohydrates (14). We interpreted these findings to indicate that glycogen was being synthesized in PTG-overexpressing cells from amino acids present in the culture medium. The current study provides insight into a potential mechanism facilitating this process, in that diversion of gluconeogenic carbon into glycogen may be partially explained by the $70 \%$ decline in expression of the catalytic subunit of G6Pase in PTG-overexpressing animals after OGTT. However, future studies, perhaps involving infusion of a gluconeogenic precursor such as $\left[{ }^{13} \mathrm{C}\right]$ alanine into animals with hepatic overexpression of PTG, will be required to determine if gluconeogenic rate has actually been altered and whether glycogen is being derived from exogenous or endogenous (e.g., intrahepatic) carbon sources. Such studies may also help to explain why blood glucose concentrations were not different or only mildly reduced in PTG-overexpressing animals relative to controls in either the fasted or fed states.

The failure to deplete glycogen in the fasted state in PTG-overexpressing animals introduces a note of caution 
in terms of considering this protein as a therapeutic target for increasing the efficiency of hepatic glucose disposal. For example, it is possible that hypoglycemic episodes would occur with longer periods of fasting or bouts of exercise in PTG-overexpressing animals. One potential approach to resolving this problem is to use another member of the glycogen-targeting subunit family that is more responsive to changes in physiological states. In addition to PTG, which is expressed in a wide variety of tissues, other members of the glycogen-targeting subunit gene family include $G_{L}$, which is expressed primarily in liver (10), and $\mathrm{G}_{\mathrm{M}}$, which is expressed primarily in skeletal muscle and heart $(9,13)$. $G_{M}$ is phosphorylated on serine46 in response to insulin (referring to the human $G_{M}$ sequence), and this appears to enhance the capacity of the $\mathrm{G}_{\mathrm{M}}-\mathrm{PP} 1$ complex to dephosphorylate and activate glycogen synthase, whereas $\beta$-adrenergic agonists cause phosphorylation of serine- 65 , resulting in dissociation and inactivation of the $\mathrm{G}_{\mathrm{M}}-\mathrm{PP} 1$ complex (29). $\mathrm{G}_{\mathrm{L}}$ lacks serine46 , and although it contains a serine at position 65 , it appears not to be regulated by a phosphorylation/dephosphorylation mechanism but is instead allosterically regulated by binding to phosphorylase a (10). Neither the hormonal or allosteric mechanisms appear to be operative in PTG $(11,12)$. Thus, further studies will be required to test the idea that expression of $G_{M}$ or $G_{L}$ might provide the benefit of improved glucose disposal in the postprandial state, without inappropriate sequestration of glycogen during fasting.

Encouragement for continuing this line of investigation comes from consideration of the relative lack of impact of PTG overexpression on steady-state levels of circulating metabolites and hormones (Table 2). In particular, in comparison to our previous report on the metabolic impact of glucokinase overexpression in liver (6), PTG expression improves glucose tolerance in normal rats without increasing circulating FFA or TG. Thus, the notion that PTG, and potentially other members of the glycogen-targeting subunit gene family, can enhance glucose uptake by specifically diverting glucose into glycogen is supported by the studies described here. Better understanding of the activities of different members of the glycogen-targeting subunit gene family will pave the way for future studies that test the utility of these molecules for lowering of blood glucose in diabetes.

\section{Acknowledgments}

The authors thank A.D. Sherry and R. Unger for critical review of the manuscript. The work was supported by a grant from the National Institutes of Health (P50H2598801 to C.B. Newgard).

1. Magnusson, I., Rothman, D.L., Katz, L.D., Shulman, R.G., and Shulman, G.I. 1992. Increased rate of gluconeogenesis in type II diabetes mellitus. A ${ }^{13} \mathrm{C}$ nuclear magnetic resonance study.J. Clin. Invest. 90:1323-1327.

2. Cline, G.W., Rothman, D.L., Magnusson, I., Katz, L.D., and Shulman, G.I 1994. ${ }^{13} \mathrm{C}$-nuclear magnetic resonance spectroscopy studies of hepatic glucose metabolism in normal subjects and subjects with insulin-dependent diabetes. J. Clin. Invest. 94:2369-2376.

3. Velho, G., et al. 1996. Impaired hepatic glycogen synthesis in glucokinasedeficient (MODY-2) subjects. J. Clin. Invest. 98:1755-1761.
4. Antinozzi, P., Berman, H.K., O’Doherty, R.M., and Newgard, C.B. 1999 Metabolic engineering with adenoviral vectors. Annu. Rev. Nutr. 19:511-514 5. Trinh, K., O'Doherty, R., Anderson, P., Lange, A.J., and Newgard, C.B. 1998 Perturbation of fuel homeostasis caused by overexpression of the glucose6-phosphatase catalytic subunit in liver of normal rats. J. Biol. Chem. 273:31615-31620

6. O’Doherty, R.M., Lehman, D., Telemaque-Potts, S., and Newgard, C.B. 1999 Metabolic impact of glucokinase overexpression in liver: lowering of blood glucose in fed rats is accompanied by hyperlipidemia. Diabetes. 48:2022-2027.

7. Ferre, T., Pujol, A., Efren, R., Bosch, F., and Valera, A. 1996. Correction of diabetic alterations by glucokinase. Proc. Natl. Acad. Sci. USA. 93:7225-7230.

8. Hariharan, N., et al. 1997. Expression of human hepatic glucokinase in transgenic mice liver results in decreased glucose levels and reduced body weight. Diabetes. 46:11-16

9. Tang, P.M., Bondor, J.M., Swiderek, K.M., and DePoali-Roach, A.P. 1991 Molecular cloning and expression of the regulatory (RG1) subunit of the glycogen-associated protein phosphatase. J. Biol. Chem. 266:15782-15789.

10. Doherty, M.J., Moorhead, G., Morrice, N., Cohen, P., and Cohen, P.T.W. 1995. Amino acid sequence and expression of the hepatic glycogen-binding (GL)-subunit of protein phosphatase-1. FEBS Lett. 375:294-298.

11. Doherty, M.J., Young, P.R., and Cohen, P.T.W. 1996. Amino acid sequence of a novel protein phosphatase 1 binding protein (R5) which is related to the liver- and muscle-specific glycogen binding subunits of protein phosphatase 1. FEBS Lett. 399:339-343.

12. Printen, J.A., Brady, M.J., and Saltiel, A.R. 1997. PTG, a protein phosphatase 1-binding protein with a role in glycogen metabolism. Science. 275:1475-1478

13. Armstrong, C.G., Browne, G.J., Cohen, P., and Cohen, P.T.W. 1997. P1R6, a novel member of the family of glycogen-targeting subunits of protein phosphatase 1. FEBS Lett. 418:210-214.

14. Berman, H.K., O'Doherty, R.M., Anderson, P., and Newgard, C.B. 1998. Overexpression of protein targeting to glycogen (PTG) in rat hepatocytes causes profound activation of glycogen synthesis independent of normal hormoneand substrate-mediated regulatory mechanisms. J. Biol. Chem. 273:26421-26425.

15. Doherty, M.J., Cadefau, J., Stalmans, W., Bollen, M., and Cohen, P.T.W. 1998. Loss of the hepatic glycogen-binding subunit $(\mathrm{GL})$ of protein phosphatase 1 underlies deficient glycogen synthesis in insulin-dependent diabetic rats and in adrenalectomized starved rats. Biochem. J. 333:253-257.

16. Herz, J., and Gerard, R.D. 1993. Adenovirus-mediated transfer of low density lipoprotein lipase receptor gene acutely accelerates cholesterol clearance in normal mice. Proc. Natl. Acad. Sci. USA 90:2812-2816.

17. Becker, T., et al. 1994. Use of recombinant adenovirus for metabolic engineering. Methods Cell Biol. 43:161-189.

18. Buettner, R., Newgard, C.B., Rhodes, C.J., and O’Doherty, R.M. 2000. Correction of diet-induced leptin and insulin resistance via moderate elevation of plasma leptin. Am. J. Physiol. In press.

19. Jensen, J., Serup, P., Karlsen, C., Nielsen, T.F., and Madsen, O.D. 1996. mRNA profiling of rat islet tumors reveals $\mathrm{Nkx} 6.1$ as a beta-cell-specific homeodomain transcription factor. J. Biol. Chem. 271:18749-18758.

20. Newgard, C.B., Hirsch, L., Foster, D.W., and McGarry, J.D. 1983. Studies on the mechanism by which exogenous glucose is converted into liver glycogen in the rat: a direct or an indirect pathway? J. Biol. Chem. 258:8046-8052.

21. Jones, J.G., Carvalho, R.A., Franco, B., Sherry, A.D., and Malloy, C.R. 1998. Measurement of hepatic glucose output, Krebs cycle and gluconeogenic fluxes by NMR analysis of plasma glucose. Anal. Biochem. 263:39-45.

22. Brainard, J.R., Downey, R.S., Bier, D.M., and London, R.E. 1989. Use of multiple ${ }^{13} \mathrm{C}$-labeling strategies and ${ }^{13} \mathrm{C}$ NMR to detect low levels of exogenous metabolites in the presence of large endogenous pool: measurement of glucose turnover in a human subject. Anal. Biochem. 176:307-312.

23. Katz, J., Lee, W.N.P., Wals, P.A., and Bergner, E.A. 1989. Studies of glycogen synthesis and the Krebs cycle by mass isotopomer analysis with $\left[\mathrm{U}-{ }^{13} \mathrm{C}\right] \mathrm{glu}-$ cose in rats. J. Biol. Chem. 264:12994-13001.

24. Liu, Z., Barrett, E.J., Dalkin, A.C., Zwart, A.D and Chou, JY 1994. Effect of acute diabetes on rat hepatic glucose-6-phosphatase activity and its messenger RNA level. Biochem. Biophys. Res. Commun. 205:680-686.

25. Massillon, D., Barzilai, N., Chen, W., Hu, M., and Rossetti, L. 1996. Glucose regulates in vivo glucose-6-phosphatase gene expression in the liver of diabetic rats. J. Biol. Chem. 271:9871-9874.

26. Argaud, D., Kirby, T.L., Newgard, C.B., and Lange, A.J. 1997. Glucose stimulation of glucose-6-phosphatase gene expression in primary hepatocytes and Fao hepatoma cells. Requirement for glucokinase expression. J. Biol. Chem. 272:12854-12861.

27. Unger, R.H. 1981. The milieu interieur and the islets of Langerhans. Diabetologia. 20:1-11.

28. McGarry, J.D., Kuwajima, M., Newgard, C.B., Foster, D.W., and Katz,J. 1986. From dietary glucose to liver glycogen: the full circle round. Annu. Rev. Nutr. 7:51-73.

29. Dent, P., Lavoinne, A., Nakielny, S., Watt, P., and Cohen, P. 1990. The molecular mechanism by which insulin stimulates glycogen synthesis in mammalian skeletal muscle. Nature. 348:302-308. 